\title{
Zone plate focused soft X-ray lithography
}

\author{
Adam F.G. Leontowich • Adam P. Hitchcock
}

Received: 6 December 2010 / Accepted: 9 December 2010 / Published online: 29 January 2011

(C) Springer-Verlag 2011

\begin{abstract}
The zone plate focused soft X-rays of a scanning transmission X-ray microscope have been used to pattern poly(methyl methacrylate) and poly(dimethylglutarimide) films by a direct write method which is analogous to lithography with a focused electron beam. The lithographic characteristics of both polymers have been determined for $300 \mathrm{eV}$ X-rays. With low doses (1 MGy), developed lines $40 \pm 5 \mathrm{~nm}$ wide were created in poly(methyl methacrylate). At higher doses an exposure spreading phenomenon substantially increases the lateral dimensions of the developed patterns. The spreading mechanism has been identified as the point-spread function of the zone plate lens. The performance of focused soft X-ray lithography is compared to other direct write methods. The practicality of a dedicated focused soft X-ray writer instrument is discussed.
\end{abstract}

\section{Introduction}

The radiation based lithography process was first disclosed in 1852 [1]. Continuous improvement of radiation based lithography techniques has played a central role in facilitating the fabrication of ever shrinking devices (electronic [2], nanofluidic [3], photonic [4], mechanical [5], optical [6]) with ever superior performance which modern society is increasing reliant upon. Yet after more than 150 years of innovation the fundamental steps have not changed: a substrate is coated with a layer of radiation sensitive material, termed

A.F.G. Leontowich · A.P. Hitchcock $(\bowtie)$

Brockhouse Institute for Materials Research, McMaster

University, Hamilton, L8S 4M1 ON, Canada

e-mail: aph@mcmaster.ca

Fax: +1-905-5212773 the resist. Radiation is directed upon the resist in a controlled manner which causes changes to its chemical properties, patterning it into areas of irradiated and non-irradiated material. The sample then undergoes a development procedure, often accomplished by immersing the sample in a suitable solvent. The chosen solvent may remove the irradiated resist, leaving behind the non-irradiated material (positive mode), or the solvent may remove the non-irradiated resist, leaving behind the irradiated material (negative mode). After development, the desired pattern exists as a mask of resist on substrate. Development of the patterned resist is a crucial step toward actual device fabrication.

There are at least three general methods to arbitrarily pattern resists via radiation.

(1) Position a patterned mask with transparent and opaque areas between a radiation source and the resist, and flood the mask with radiation [7]. Current state of the art tools for mass production of electronic devices such as microprocessors employ the patterned mask method and use $193 \mathrm{~nm}$ photons to produce sub-50 $\mathrm{nm}$ features on an industrial scale [8].

(2) Position a patterned reflecting surface between the radiation source and the resist, irradiate this surface, and allow only the reflected radiation to impinge upon the resist [9]. It is anticipated that this projection method with extreme ultraviolet photons $(13.5 \mathrm{~nm})$ will supersede the pattern mask method for industrial scale production at the $15 \mathrm{~nm}$ node.

(3) Focus radiation with optics or electromagnetic fields to a small point, then scan either the beam above the resist, or the resist below the beam, in a controlled manner. This is the direct write method.

Both patterned mask and projection methods rely on the direct write method to create the arbitrarily patterned masks 
and surfaces for micron and sub-micron lithography. Focused beams are also generally used for low volume production when the cost of fabricating a mask is prohibitive, such as producing masters for microfluidic devices, prototyping, and in fabricating one-off devices.

Reports of sub-100 nm direct write patterning and lithography with focused photons are scarce relative to those involving focused electrons and ions. We hypothesize several reasons for this. The first report of patterning with a focused electron beam (1960) demonstrated sub-100 nm features [10]. For far field techniques, the Rayleigh criterion prevents focusing of photons at visible and UV wavelengths into sub-100 nm spots (though sub-100 nm direct write patterning with visible light and highly specific resists has been reported [11]). As one reaches beyond the deep ultraviolet in search of higher resolution, massive challenges arise in optics fabrication: almost negligible differences in refractive index from unity, relatively large absorption coefficients, and the necessity to fabricate optical elements with an accuracy comparable to the wavelength of X-rays [12]. Another early problem was the unavailability of bright X-ray sources. Meanwhile, very early in the history of electron beam lithography, electron beams could be focused into sub-10 nm Rayleigh resolution spots with adequate current to expose resist materials. By 1980 there were commercially available electron beam writer systems, and focused ion beams had demonstrated sub-100 nm patterning capability [13]. An early comparison of direct write electron and UV systems reported that focused electron beams had greater write speed and could produce narrower features [14]. In addition to the massive technological challenges that needed to be overcome at that time, it was not clear that a focused photon source of any energy could provide any advantages over a focused electron beam for sub-100 nm direct write patterning. At present, "X-ray lithography" is nearly synonymous with the patterned mask method and broadband synchrotron radiation, while direct write electron beam systems are the exposure tools of choice in terms of minimum feature size and flexibility.

Spurred by the developers of water window $(2.5-4.5 \mathrm{~nm})$ soft X-ray microscopy $[15,16]$ the technological barriers hindering sub-100 $\mathrm{nm}$ direct write patterning with focused $\mathrm{X}$-rays have since been overcome. Modern scanning transmission X-ray microscopes (STXM) [17-19] routinely focus monochromatic soft X-rays into sub-40 nm Rayleigh resolution spots using Fresnel zone plates [20] (fabricated by electron beam lithography!) with the state of the art resolution being $10 \mathrm{~nm}$ [21]. Though the efficiencies of zone plates are quite low (often less than 10\%) the performance of modern third generation synchrotrons and associated beamlines is so tremendous that instrument performance is rarely flux limited. The first example of direct write X-ray patterning was reported by Zhang et al. [22], who used focused
$317 \mathrm{eV}$ soft X-rays at the National Synchrotron Light Source (NSLS) to pattern poly(methyl methacrylate). Larciprete et al. [23] used focused $640 \mathrm{eV}$ soft X-rays at ELETTRA to pattern LiF films. Wang et al. [24, 25] using Advanced Light Source STXM 5.3.2.2 (formerly referred to as 5.3.2) have pioneered direct write $\mathrm{X}$-ray patterning at multiple wavelengths to pattern multi-layer polymer films with chemical selectivity.

However, in all reported direct write X-ray patterning experiments to date the best minimum feature size of either undeveloped or developed patterns is only $90 \pm 14 \mathrm{~nm}$ in hydrogen silsesquioxane (HSQ) [26], despite writing with focused X-ray sources of much finer spot size. The resolution limitation is due to an exposure spreading phenomenon whose origin has not been understood until this work. Here we report the creation of patterns at $40 \pm 5 \mathrm{~nm}$ feature size in thin layers of polymeric resists with focused soft X-rays, their development, and subsequent characterization. Feature broadening at higher doses is documented and the exposurespread mechanism is shown to be due to the point-spread function of the zone plate lens. The performance relative to other radiation based lithography techniques is discussed and the potential for practical applications of focused soft $\mathrm{X}$-ray lithography is addressed.

\section{Experiment}

\subsection{Materials}

Poly (methyl methacrylate) (PMMA) $M_{w}: 315000 M_{w} / M_{n}$ : 1.05 electronics grade was purchased from Polymer Source Inc. Toluene $99.9 \%$ Chromasolv ${ }^{\circledR}$, 4-methyl-2-pentanone (MIBK) $>98.5 \%$ ACS reagent grade, and $N, N$-dimethylformamide (DMF) 99.9\% Chromasolv® were purchased from Sigma-Aldrich. 2-Propanol (IPA) $99.5 \%$ and water HPLC grade were purchased from Caledon. Mica was purchased from Ted Pella Inc. $75 \mathrm{~nm} \mathrm{Si}_{3} \mathrm{~N}_{4}$ windows $(1 \times 1 \mathrm{~mm}$ window area in a $5 \times 5 \mathrm{~mm} \mathrm{Si}$ wafer frame) were purchased from Norcada Inc. Poly (dimethylglutarimide) (PMGI) was a gift from Professor Ash Parameswaran, Simon Fraser University, British Columbia, Canada.

\subsection{Complementary characterization}

Atomic force microscopy (AFM) measurements were performed with a Quesant 350 microscope equipped with Budget Sensors Multi75Al probes. All images were collected with the AFM operating in non-contact mode at a $0.5 \mathrm{~Hz}$ scan rate with $512 \times 512$ pixels. Scanning electron microscope (SEM) measurements were performed with a JEOL JSM-7000F microscope operating at $10 \mathrm{keV}$ with a $60 \mu \mathrm{A}$ beam current at a working distance of $5 \mathrm{~mm}$. A Gatan model 
682 Precision Etching Coating System was used to coat all samples with $5 \mathrm{~nm}$ Pt before analysis. AFM measurements were always performed before Pt coating and SEM measurement to avoid artifacts due to Pt deposition or electron beam damage.

\subsection{Preparation of thin polymer films on $\mathrm{Si}_{3} \mathrm{~N}_{4}$ substrates}

Thin polymer films were fabricated by spin casting four drops of a $1.0 \% \mathrm{w} / \mathrm{w}$ PMMA/toluene solution, or a $2.8 \%$ w/w PMGI/DMF solution onto a $1.5 \times 1.5 \mathrm{~cm}$ piece of freshly cleaved mica. The films remained in ambient air for 10 minutes and were then cut into $3 \times 3 \mathrm{~mm}$ pieces with a scalpel. Upon slowly dipping the mica into a Petri dish filled with water, small pieces of the film release and float on the surface, which were then caught on $\mathrm{Si}_{3} \mathrm{~N}_{4}$ windows. The pieces were positioned such that the polymer film only partially covered the window to allow for measurements of the spectrum of incident radiation $\left(I_{o}\right)$. The samples were then annealed for 1 hour at $140^{\circ} \mathrm{C}$ (PMMA) or $230^{\circ} \mathrm{C}$ (PMGI) at reduced pressure $\left(\sim 2 \times 10^{-2}\right.$ Torr $)$. The annealed films were consistently $45 \pm 5 \mathrm{~nm}$ (PMMA) and $65 \pm 5 \mathrm{~nm}$ (PMGI) as measured by AFM and near edge X-ray absorption fine structure (NEXAFS) spectroscopy.

\subsection{Scanning transmission X-ray microscope and patterning}

An interferometrically controlled STXM [17] on bend magnet beamline 5.3.2.2 [27] at the Advanced Light Source (ALS) at Lawrence Berkeley National Lab (LBNL) was used to direct write the desired patterns. The zone plate lens ( $25 \mathrm{~nm}$ outer zone, $240 \mu \mathrm{m}$ diameter, $90 \mu \mathrm{m}$ central stop) was supplied by the Center for X-ray Optics (CXRO), LBNL. A $\mathrm{N}_{2}$ gas filter (a differentially pumped $1 \mathrm{~m}$ long section of the beamline at a pressure of $\sim 0.6$ Torr) was used to eliminate higher order radiation. The patterning was carried out with the STXM tank backfilled with He to $1 / 3$ atmospheric pressure, after evacuation of air. A uniform area of the film was located and a sharp focus was established at a point within $80 \mu \mathrm{m}$ of the area to be patterned. Patterning was executed using the pattern generation capabilities of the STXM Control software, as described by Wang et al. [24]. Special care must be taken to ensure that the patterning is done on a pristine area of the film just beside the area of focusing, since even very brief (1 ms) exposures used while imaging are sufficient to cause unwanted patterning, especially to PMMA. The patterned areas were never imaged by STXM before or after patterning. For all experiments the exposure energy was fixed at $300 \mathrm{eV}$. The detector efficiency in the $\mathrm{C} \mathrm{K}$ edge region [17], mass loss, and other instrumental parameters have been taken into account in computing the absorbed radiation doses presented, which are believed to be precise within $10 \%$, using methods described in detail earlier [24, 25].

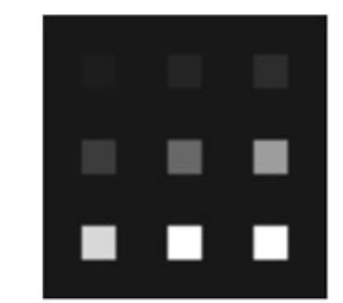

$\mathbf{a}$

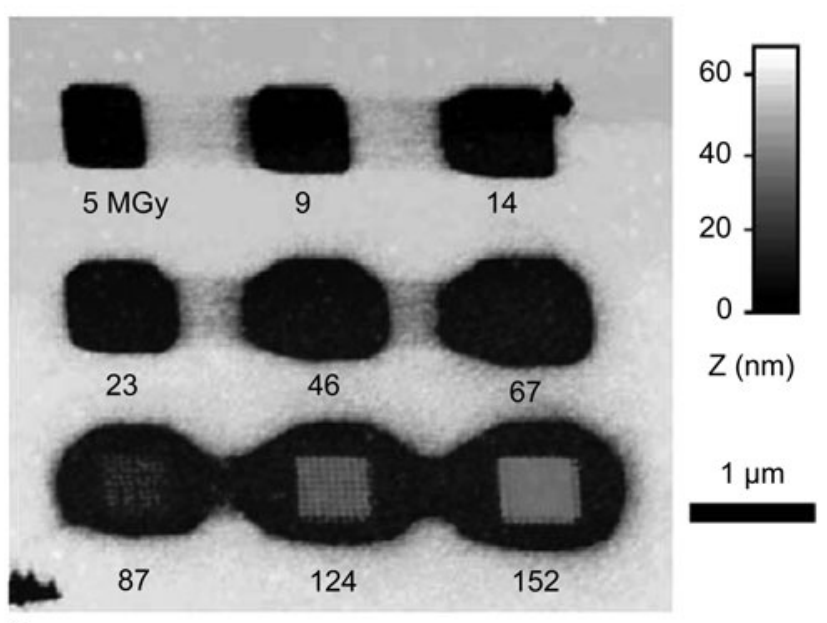

c

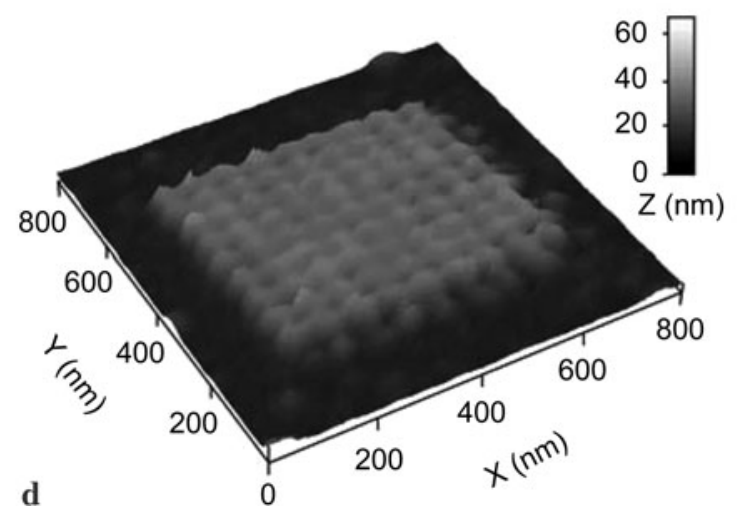

Fig. 1 (a) The pattern used to drive the sample positioning stages relative to the focal point consists of nine $600 \times 600 \mathrm{~nm}$ areas. (b) Each square in (a) is composed of $10 \times 10$ individual exposures spaced $60 \mathrm{~nm}$ center to center. (c) AFM image of the pattern directly written in PMMA with focused $300 \mathrm{eV}$ monochromatic X-rays after development. (d) Expanded three dimensional view of the eighth area in (c). The measured center to center spacing of the crosslinked PMMA mounds is $60 \pm 5 \mathrm{~nm}$

\subsection{Development of patterned polymer films}

Development of PMMA was accomplished by immersing the entire $\mathrm{Si}_{3} \mathrm{~N}_{4}$ window in 3:1 IPA:MIBK for $30 \mathrm{~s}$, then IPA for $15 \mathrm{~s}$. PMGI was developed by immersing the entire $\mathrm{Si}_{3} \mathrm{~N}_{4}$ window in 7:3 IPA: $\mathrm{H}_{2} \mathrm{O}$ for $30 \mathrm{~s}$, following the procedure of Johnstone et al. [28]. All development occurred at ambient temperature $\left(\sim 20^{\circ} \mathrm{C}\right)$. 


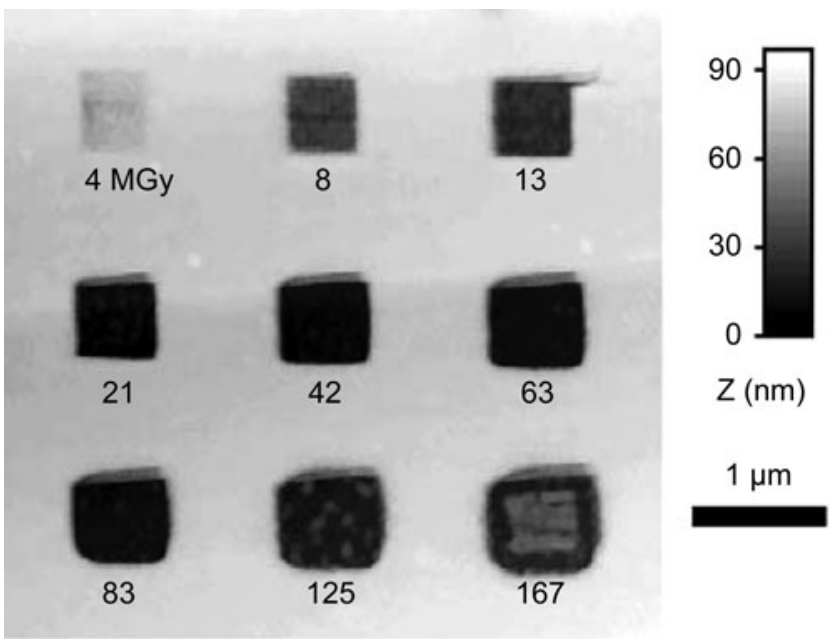

Fig. 2 AFM image of the pattern directly written in PMGI with focused $300 \mathrm{eV}$ monochromatic $\mathrm{X}$-rays after development (the resolution of this image is equal to Fig. 1c)

\section{Results}

\subsection{Lithographic characteristics of PMMA and PMGI patterned with $300 \mathrm{eV}$ soft X-rays}

PMMA was exposed to a pattern consisting of nine $600 \times$ $600 \mathrm{~nm}$ exposure areas (Fig. 1a) which was designed to efficiently characterize the lithographic transitions of PMMA. We define two lithographic transitions: (a) the dose/dose range required for complete removal of the resist from the exposure area after development (positive mode), and (b) the dose/dose range required to transition from positive mode to negative mode. Each square is composed of $10 \times 10$ pixels spaced $60 \mathrm{~nm}$ center to center (Fig. 1b). The pixel dwell time of the exposure areas increases from left to right and top to bottom such that each area receives a controlled dose that progressively increases from one area to the next. After patterning the sample was removed from the STXM and developed. The developed pattern was then imaged with AFM (Fig. 1c) and SEM (not shown). A high resolution AFM image of the eighth area (Fig. 1d) shows that all $10 \times 10$ exposed points are resolved as mounds of crosslinked (negative mode) PMMA with a center to center spacing of $60 \pm 5 \mathrm{~nm}$.

Many areas of the PMMA film were patterned covering a dose range from 0.1-300 MGy. The dose dependent lithographic transitions of PMMA were determined from analysis of AFM images of these features and the observations are summarized in Table 1.

The dose dependent lithographic transitions of PMGI were measured in the same fashion. Patterned areas were prepared by controlled exposure to $300 \mathrm{eV}$ focused X-rays, developed and imaged with AFM (Fig. 2). The lithographic transitions of PMGI were determined from analysis of the

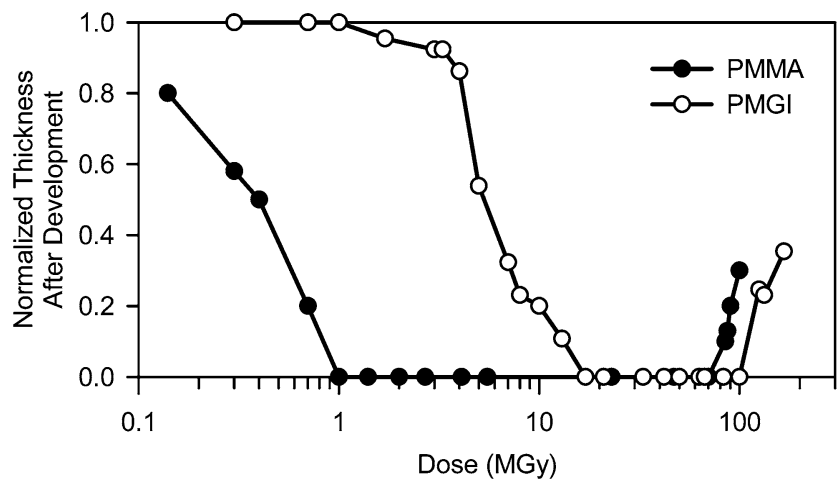

Fig. 3 Plot of residual thickness, as determined by AFM after development, as a function of dose for PMMA and PMGI exposed to $300 \mathrm{eV}$ monochromatic X-rays. 1 corresponds to the initial spun cast thickness, 0 being full removal

AFM images and the observations are summarized in Table 2. The response of both polymers to dose after development was quantified by AFM and is presented graphically in Fig. 3.

\subsection{Minimum developed feature size}

An elbow pattern (Fig. 4a) was directly written in a PMMA film with a dose of $1 \mathrm{MGy}$. After patterning the sample was removed from the STXM, developed, and imaged with AFM (Fig. 4b). The developed lines were determined by AFM to be $\sim 50 \mathrm{~nm}$ deep (Fig. 4c), indicating that these lines extend down to the substrate. The SEM image of the developed elbow pattern (Fig. 4d) reveals a different surface morphology at the center of the lines versus other areas of the film. The morphology at the center of the lines is identical to that of bare $\mathrm{Si}_{3} \mathrm{~N}_{4}$, again confirming complete removal of PMMA from the exposure area after development. We determined the width of the developed horizontal lines to be $40 \pm 5 \mathrm{~nm}$ with a pitch of $120 \mathrm{~nm}$ (a 1:2 linewidth-to-spacewidth ratio) by analyzing the SEM image. The width of the vertical lines is somewhat larger and less regular which we suspect is due to incomplete closure of the shutter between pixels and the direction in which the patterns are written (horizontal line after horizontal line from bottom to top). The minimum 1:1 linewidth achievable, as well as the minimum negative mode linewidth, were determined by adjusting the size of the elbow pattern and were characterized in the same fashion. Minimum linewidth values for PMMA are presented in Table 3.

\subsection{Exposure spreading phenomenon}

While attempting to find the optimum doses for positive mode lithography it was readily apparent that the width of the developed features increased with increasing dose. To demonstrate, several identical elbow patterns were written 
Table 1 Lithographic characteristics of PMMA exposed to $300 \mathrm{eV}$ soft X-rays

\begin{tabular}{ll}
\hline Dose (MGy) & Observation \\
\hline$<1$ & Incomplete removal of irradiated material \\
$1-85$ & Complete removal of irradiated material (positive mode) \\
$85-95$ & Onset of crosslinking (negative mode) \\
$95-150$ & Fine crosslinked features \\
$>150$ & Extensive crosslinked features \\
\hline
\end{tabular}

Table 2 Lithographic characteristics of PMGI exposed to $300 \mathrm{eV}$ soft X-rays

\begin{tabular}{ll}
\hline Dose (MGy) & Observation \\
\hline$<15$ & Incomplete removal of irradiated material \\
$15-110$ & Complete removal of irradiated material (positive mode) \\
$110-140$ & Onset of crosslinking (negative mode) \\
$>150$ & Extensive crosslinked features \\
\hline
\end{tabular}

directly in PMMA differing only in dose. After patterning, the sample was removed from the STXM, developed, and imaged with SEM. The series of SEM images (Fig. 5) clearly shows that as dose increases, the width of developed features increases. Closely spaced features experience substantial distortion; at higher doses the exposure spread can cause closely spaced lines to merge together. In Fig. 5d the exposure areas received sufficient dose to be in the negative mode regime; the 1:1 lines of crosslinked PMMA are $90 \pm 5 \mathrm{~nm}$ wide. Surprisingly, material was removed more than $300 \mathrm{~nm}$ away from the expected exposure area. It is this type of exposure-spread phenomenon that has limited the minimum achievable feature sizes for direct write X-ray patterning in the past [23-26]. Understanding the origin, which in turn could lead to strategies to minimize the spread, was a major goal of this research.

Two single pixel exposures were made in PMMA with relatively high doses (370 MGy) to investigate the exposurespread phenomenon. After patterning the sample was removed from the STXM, developed, and imaged by AFM (Fig. 6a). This image shows that the spatial distribution of the exposure spread around a single pixel exposure is anisotropic and extends several hundred nm from the central exposure point. The second single pixel exposure made under identical conditions has an identical spatial distribution. The spatial distribution of the apparent exposure spread around multiple separated single pixel exposures is completely reproducible with fine detail in PMMA. The developed single pixel exposures were also imaged by SEM (Fig. 6b). The spatial distribution observed by SEM is identical to that measured by AFM (Fig. 6a), negating AFM tip convolution effects as the origin of the reproducible anisotropic pattern.

The same single pixel, high dose exposure experiment was performed in PMGI. The developed patterns were imaged with AFM (not shown) and SEM (Fig. 6c). AFM im-
Table 3 Minimum developed feature widths created in PMMA

\begin{tabular}{ll}
\hline Feature Classification & Width (nm) \\
\hline Positive mode 1:2 lines & $40 \pm 5$ \\
Positive mode 1:1 lines & $75 \pm 5$ \\
Negative mode 1:1 lines & $90 \pm 5$ \\
\hline
\end{tabular}

ages were not included because the features observed were identical to those in the SEM images. The spatial distribution of the exposure spread around multiple separated single pixel exposures is anisotropic and reproducible with fine detail in PMGI. A comparison of Figs. $6 \mathrm{~b}$ and $6 \mathrm{c}$ shows the overall shape of the anisotropic spatial distribution is independent of the resist.

\section{Discussion}

4.1 Focused soft X-ray lithography in PMMA and PMGI at $300 \mathrm{eV}$

\subsubsection{Lithographic characteristics of PMMA and PMGI for 300 eV monochromatic $X$-rays}

There are hundreds of reports of radiation based lithography involving PMMA but relatively few involving PMGI. Our measured dose value of $1 \mathrm{MGy}$ to adequately expose PMMA for positive mode lithography (Table 1) is consistent with other reported values (0.85 MGy [5], 1.4 MGy [29], 0.42 MGy [30]). However, caution must be used when comparing this value, as well as all dose values compiled in Tables 1 and 2, to other studies involving PMMA and PMGI, as these values also depend critically upon certain development (composition, development and rinse time, temperature) and 


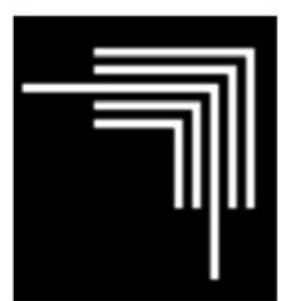

$\mathbf{a}$

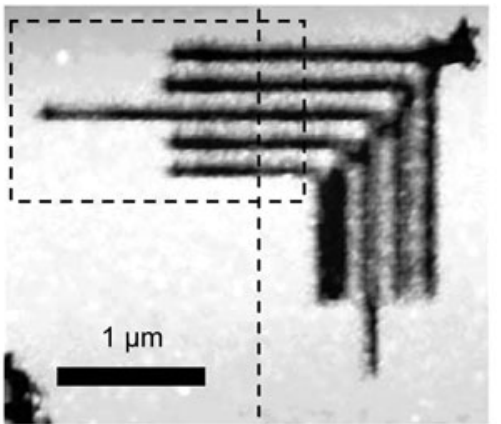

b
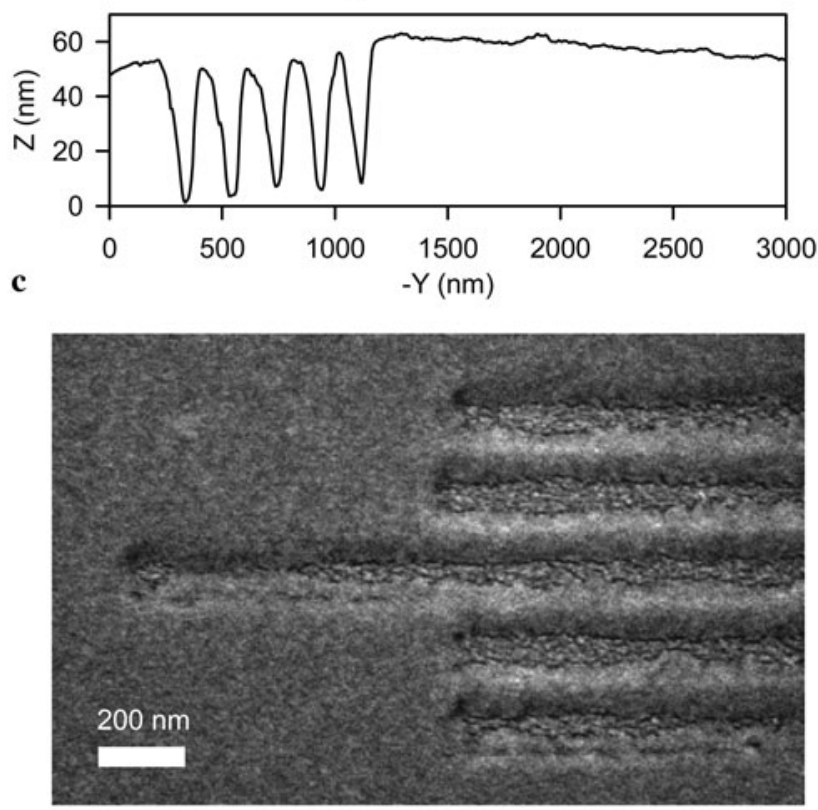

d

Fig. 4 (a) The elbow pattern used to drive the sample positioning stages relative to the focal point. (b) AFM image of the pattern directly written in PMMA with focused $300 \mathrm{eV}$ monochromatic X-rays after development. Dose: $1 \mathrm{MGy}$. (c) Line out from the dashed vertical line in (b). (d) SEM image of the region outlined by the dashed rectangle in (b) demonstrating $40 \pm 5 \mathrm{~nm} \mathrm{1:2} \mathrm{developed} \mathrm{lines}$

resist ( $M_{w}$ and $M_{n}$, casting solvent, thermal treatment) details [31,32]. Still, we note that even though the wavelengths between these studies are very different (and thus primary radiation effects are different), a similar dose for full removal was determined, which suggests that secondary radiation damage processes dominate, and the overall process is mainly related to total energy deposited per unit of material (i.e. absorbed dose). We find it difficult to avoid causing a measurable change to the developed height of PMMA, even with the shortest possible dwell times $(\sim 1 \mathrm{~ms}$ due to the time needed to open and close an in-vacuum piezo shutter) and very narrow entrance and exit slit settings (thus minimizing the photon flux). The transition from non-irradiated height to full removal inside the exposure area is very rapid and occurs over a dose range of less than $1 \mathrm{MGy}$ (Fig. 3). On the other hand the positive mode response of PMGI (Fig. 3)
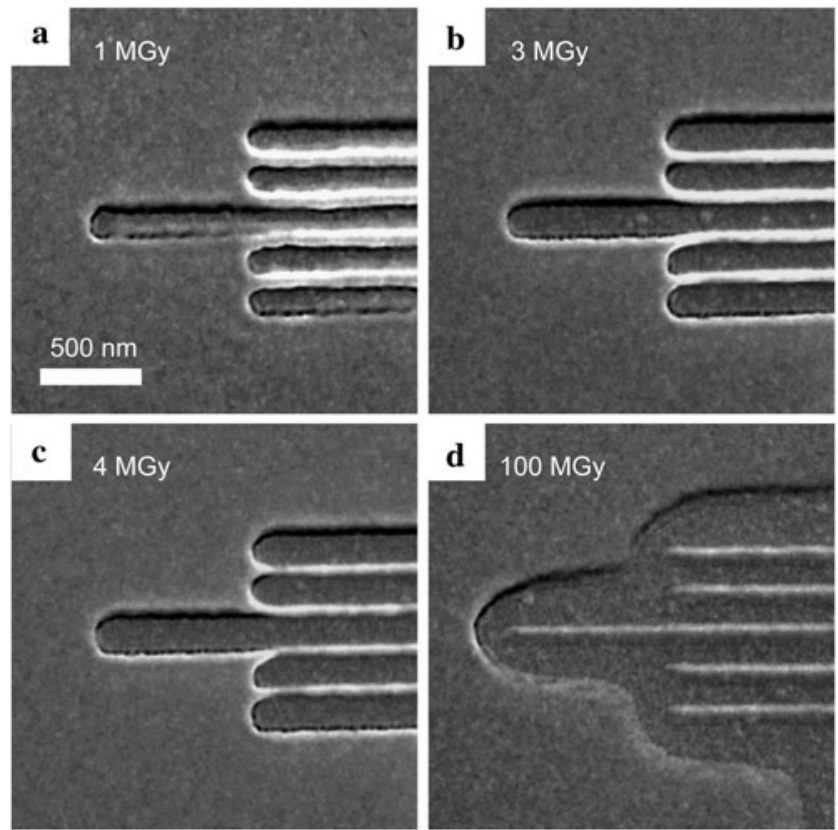

Fig. 5 SEM images of identical elbow patterns directly written in PMMA with focused $300 \mathrm{eV}$ monochromatic X-rays after development (all images are the same scale). (a) An optimum dose of 1 MGy produces the narrowest positive mode features. The developed linewidth increases with increasing dose (b), 3 MGy, (c) 4 MGy. (d) An extensive positive mode region extends beyond the high dose (100 MGy) negative mode regions. The crosslinked 1:1 lines of (d) are $90 \pm 5 \mathrm{~nm}$ wide

is much more gradual. It takes much more dose to cause a thickness change to PMGI as compared to PMMA, and the last few nm of PMGI are especially difficult to remove. More dose is required in order to fully remove all material down to the substrate. Consequently, the feature sizes broaden, and so the developed resolution of PMGI is not as high as PMMA.

PMMA and PMGI are somewhat unique in that they behave as positive resists at low doses and negative resists at high doses, as demonstrated in Figs. 1c and 2. This dose dependent transition from positive to negative mode has been known for some time for PMMA [2], but we are unaware of any reports of this behavior for PMGI. The onset of crosslinking for PMMA consistently occurred within a relatively narrow dose range (Table 1), and fine sub-100 nm features can be made with doses just beyond the onset, as demonstrated in Figs. 1d and 5d. Within the extensive crosslinking dose range the crosslinked material from individual pixels blurs together, such that the pixilation is smoothed and the thickness is even within $\pm 1 \mathrm{~nm}$ (Fig. 1c, area 9). In contrast, the onset of crosslinking in PMGI is not as well defined and occurs over a much larger dose range (Table 2). Within this dose range we observe random blobs of crosslinked PMGI with spotty coverage of the substrate (Fig. 2, area 8). The substrate could be fully covered with crosslinked PMGI using doses greater than 140 MGy, 


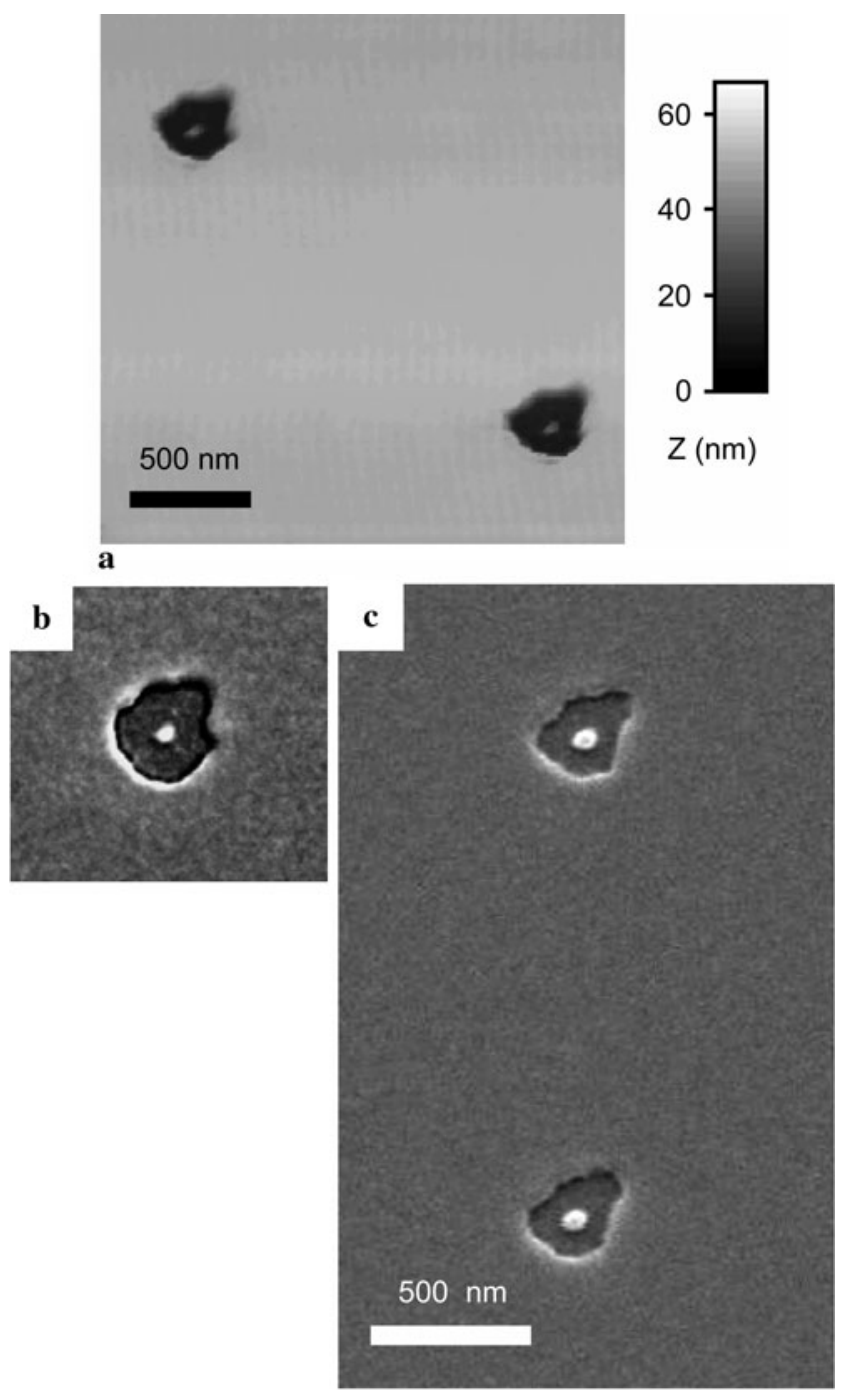

Fig. 6 (a) AFM image of a developed PMMA pattern consisting of two single pixel exposures, each at a dose of $370 \mathrm{MGy}$. The dots in the centers are crosslinked PMMA. (b) SEM image of the top left single pixel exposure of (a). (c) SEM image of a developed PMGI pattern consisting of two single pixel exposures, each at a dose of $900 \mathrm{MGy}$. The dots in the centers are crosslinked PMGI

though the thickness of this coverage was uneven at $\pm 5 \mathrm{~nm}$ (Fig. 2, area 9).

\subsubsection{Minimum feature size}

We have reduced the minimum feature sizes fabricated with focused soft X-rays by a factor of five from our earlier reports. In our previous work [24, 25, 33] films were patterned with high doses, and the patterned areas were then imaged at low doses with the STXM using photon energies sensitive to the damage chemistry (in PMMA and PMGI, $\mathrm{C}=\mathrm{O}$ bond removal). The feature sizes were determined from analysis of the STXM images. In the case of PMMA, when imaging at $288.5 \mathrm{eV}$ (the sensitive $\mathrm{C} 1 \mathrm{~s}(\mathrm{C}=\mathrm{O}) \rightarrow \pi_{\mathrm{C}=\mathrm{O}}^{*}$ transition) the contrast in each pixel of the image is proportional to the number of carbonyl bonds. As the exposure area receives more dose, more carbonyl bonds are broken. Doses below 5 MGy show negligible change in contrast for PMMA [32], so the patterned areas must receive more than $5 \mathrm{MGy}$ to just be observable above the background, which is five times that required for positive mode lithography.

The mechanism for development of PMMA is very well known [2, 34]. Briefly, PMMA experiences primarily main chain scission when exposed to ionizing radiation. Lower molecular weight (irradiated) PMMA is extremely soluble in the developer solution, whereas higher molecular weight (non irradiated) PMMA is only sparingly soluble. One main chain scission in the middle of a polymer chain will reduce its molecular weight in half, which has a very large effect on its solubility [31,34]. Thus, without development, one bond scission has a negligible effect on contrast when visualizing the patterns by X-ray microscopy, whereas, with development, one bond break can produce a very large contrast change. Furthermore, in this work we have imaged the developed patterns by AFM and SEM which have significantly better spatial resolution, in the sub-5 $\mathrm{nm}$ range, as opposed to the $30 \mathrm{~nm}$ spatial resolution of the STXM with $25 \mathrm{~nm}$ zone plates. By incorporating a development step and employing imaging techniques with higher resolution we are able to visualize very low doses and avoid the large dose and thus exposure-spread regime, which has lead to the significant improvement in minimum feature size.

We believe the $40 \pm 5 \mathrm{~nm}$ features presented here are the smallest created with a zone plate lens at any wavelength in any orientation (including demagnifying a patterned mask [35]). The zone plates used in this study achieve $30 \mathrm{~nm}$ diffraction-limited resolution, thus we have demonstrated patterning at 1.3 times the diffraction limit. Still, many variables were not optimized. Avenues toward further reduction of minimum feature size are discussed in Sect. 4.3.

\subsection{Exposure spreading mechanism for focused soft X-rays}

Previous reports of STXM mapping of chemical changes caused by focused soft X-rays have observed signs of apparent exposure beyond the expected exposure area [24, 25]. Moving to development of patterns has reduced minimum achievable feature sizes dramatically but the exposurespread phenomenon qualitatively depicted in Fig. 5 still exists. This spread is also apparent in Figs. 1 and 2-the squares become much wider than their defined $600 \times 600 \mathrm{~nm}$ exposure areas with increasing dose. The features of this exposure spreading mechanism are quantifiable - the lateral extent of the spread depends on the polymer used and dose $[24,25]$. Other direct write X-ray patterning studies have noted that this takes place not only in organic resists, but also inorganic resists [23, 26], and self assembled monolayers [36]. The radiation based lithography literature contains 
numerous examples of effects which can cause the actual exposure area to be larger than intended (i.e. "proximity effects") with very similar observable characteristics to this study, though the underlying mechanistic theories are very different. Several hypotheses have been put forward, but there is no consensus for focused soft X-rays.

Reports have shown that instability of a patterned mask or the radiation source can degrade the fidelity of patterning, blurring sharp features [37]. The possibility of unwanted movement of our focal point, which could cause the actual exposure area to be larger than anticipated, was considered but dismissed since the position of the focal point relative to the sample is stabilized by an interferometric feedback system to $\pm 10 \mathrm{~nm}$ in $\mathrm{X}, \mathrm{Y}$, and $\mathrm{Z}$ [17] with a feedback response in the $100 \mathrm{~Hz}$ range. The positional accuracy and stability of the STXM microscope is clearly demonstrated by the image presented in Fig. 1d where all 10 by 10 single pixel exposures are faithfully reproduced as dots of crosslinked PMMA spaced $60 \pm 5 \mathrm{~nm}$ apart. The beam was positioned over each pixel for $225 \mathrm{~ms}$, which is $\sim 200$ times longer than necessary for positive mode lithography in PMMA. This is a testament of the positional accuracy of ALS STXM 5.3.2.2, be it for patterning or imaging.

Observing the spread distribution surrounding single pixel exposures as revealed by development provides tremendous insight into the mechanism of exposure spread for the case of focused soft X-rays. Our PMMA and PMGI films are expected to be uniform since they were annealed above their respective $T_{g}$ 's for one hour. Therefore, one expects an isotropic circular pattern for the case of a migrating damaging species spreading outward in a uniform medium. Instead, Fig. 6a shows that the spatial distribution of damage is not isotropic. Had this only been observed in one exposure, the source of the anisotropy could be rationalized as statistical variation in the migration of a damaging species, possibly due to a non uniform film. However we observe an identical anisotropic exposure-spread distribution in AFM and SEM images of identical repeated single pixel exposures. The migration of any damaging species produced by radiation, outward from the exposure area either through the sample or above it, is reasonably expected to be a random walk. Any suspected primary scattering processes [36] or possible radiation initiated propagating chemical reactions [38] would also be random. Since the spatial distribution of damage is not random, we deduce that the exposure-spread mechanism does not involve any process with a statistically random spatial distribution. Comparison of the SEM images of the spread distribution in PMMA (Fig. 6b) and PMGI (Fig. 6c) shows that the exposure spread is the same in two different polymers, when patterned under identical conditions. Ongoing work involving similar single pixel patterning experiments executed with the STXM at the Canadian Light Source (Saskatoon, SK) produced different, reproducible anisotropic spread patterns. Since the anisotropic exposure-spread distribution is independent of the resist, it must involve something outside the sample.

The point-spread function of any given (zone plate) lens is expected to be the same for each single pixel exposure. In the best-case scenario, a perfectly fabricated lens, uniformly illuminated with coherent radiation will produce an intensity distribution that is an Airy disk in the focal plane. The focal point is not a "spot" of uniform intensity and finite diameter, but is in fact an intensity/dose distribution [39]. We have determined that a $1 \mathrm{MGy}$ exposure is required to clear PMMA from the exposed area after development. When the exposure times are increased, the dose threshold of $1 \mathrm{MGy}$ is exceeded further and further away from the central axis of the focal point, leading to the exposure-spread phenomenon. The relationship between the distance of exposure spread from the center of the focal point versus dwell time reflects the point-spread function. This approach has been used to characterize the point-spread function of zone plate lenses in two dimensions with non-ionizing radiation [40]. If the illumination or the lens itself deviates from perfection, the point-spread function will not be symmetric and the pattern developed after a single pixel exposure will mirror these deviations, leading to a non symmetric dose distribution and therefore a non symmetric exposure-spread distribution, independent of the resist.

We hypothesize that the anisotropy of the exposurespread distribution for a single pixel exposure is due to some combination of aberrations in the zone plate and/or non uniform illumination of the zone plate. At this stage we can only say that the exposure-spread mechanism for the case of patterning with zone plate focused soft X-rays is related to the instrument properties, in particular, the point-spread function of the lens. Though the diffraction-limited value for resolution (the Rayleigh resolution) is often referred to as the "spot size", this is a clear illustration of why that should not be taken literally. For example, the diameter of the first null of the Airy pattern for a zone plate with a $25 \mathrm{~nm}$ outermost zone at perfect focus is $61 \mathrm{~nm}$, and $16.2 \%$ of the total intensity lies beyond that in the best-case scenario [39]. Acknowledgement of the point-spread function in STXM is clearly important for patterning but it is also critical for spectromicroscopy where spectral signals ascribed to a very small object do in fact contain contributions from X-ray absorption outside of that small object. While this is not important for majority signals, it could be important for minority components.

There is much debate concerning the radius over which a secondary electron (or any migrating damaging species for that matter) produced within the exposure area could migrate and spread damage, and it is this distance that is thought to be a fundamental limit of lithographic techniques which employ ionizing radiation [41, 42]. In solids the radius over which a liberated secondary electron could migrate 
and cause damage can be predicted by the universal inelastic mean free path curve [43]. This distance is not likely to be more than $10 \mathrm{~nm}$ given that the maximum kinetic energy is the photon energy itself, and $300 \mathrm{eV}$ soft X-rays are used. The observed damage spread distribution surrounding single pixel exposures provides an excellent opportunity to briefly comment. Under identical conditions, the anisotropic exposure-spread distribution is completely reproducible in fine detail within the resolution of the AFM and SEM images. Therefore, we infer that the radii of any damaging secondary processes initiated by $300 \mathrm{eV}$ photons must be less than $10 \mathrm{~nm}$, for, if this were not the case, these fine details would noticeably change from exposure to exposure.

\subsection{Avenues toward reducing minimum feature size}

\subsubsection{Improving the resist and development}

PMMA was chosen as a resist for this study because it is consistently found to be one of the highest resolution resists using many different radiation sources and patterning techniques. One avenue to higher resolution would be optimization of the development procedure. The 3:1 IPA:MIBK developer system was employed due to its popularity in the literature, which should facilitate a more valid direct comparison of our results to those of others. Ultrasonic agitation methods [44], cold development [45], as well as other solvent systems $[44,46]$ have been reported to reduce the width of developed features in PMMA. Thermal treatment methods have also led to reduced minimum features sizes in PMMA [47]. Other resists such as HSQ [26] could offer higher resolution than PMMA. One can also envisage a resist and developer system designed specifically for focused soft X-ray lithography.

\subsubsection{Improving the optics and exploring new energies}

Perhaps the most obvious way to increase the resolution of any direct write technique would be to incorporate higher quality optics; patterning with improved zone plates should lead to a reduction in minimum feature size. Zone plates with $12 \mathrm{~nm}$ outer zone width, which have resolved 1:1 $12 \mathrm{~nm}$ lines, have recently been fabricated [6]. Another possibility would be to use the third order rather than first order focus of a zone plate. This operational mode is difficult to establish but it provides three times smaller Rayleigh spot size $[20,48]$. This approach has been used to resolve 1:1 $14 \mathrm{~nm}$ lines with a zone plate having $25 \mathrm{~nm}$ outer zones [49]. In principle, patterning with focused X-rays could be accomplished with Kirkpatrick-Baez (KB) X-ray focusing mirrors instead of zone plates [20]. One dimensional resolution as small as $7 \mathrm{~nm}$ has recently been achieved in a long beam lines with multiple KB focusing optics [50]. KB focusing has the added benefit of being achromatic. It may be possible to achieve lower minimum feature sizes by patterning at other soft X-ray energies. Patterning with lower energy electrons has been reported to decrease linewidths [51, 52] but to our knowledge this has not been explored for patterning with monochromatic X-rays. We note that lower electron beam energy is not intrinsically a recipe for higher resolution since the electron beam writers used to fabricate the smallest zone width zone plates, such as the LBNL Nanowriter [53], operate at $50-100 \mathrm{keV}$.

\subsection{Outlook}

Like the earliest reports of focused electron beam patterning [10], we and others have adapted existing X-ray microscopes to write patterns. Currently there are eight soft X-ray STXMs in the world and at least three more are under construction or are approved projects [54], all of which are technically capable of this type of work. Due to the requirement of strictly monochromatic X-rays for best focus with zone plates, at present STXMs are only found at high brightness third generation synchrotron light sources, for which there is high demand. These microscopes were not designed for patterning and so one faces certain instrumental limitations. For the STXM, fine focus is achieved by scanning the zone plate to sample distance and observing the transmitted images of a small opaque object. Soft X-ray absorption cross sections are relatively high, so STXM samples must be thin $(\sim 100 \mathrm{~nm}$ for illumination at the $\mathrm{C} 1 \mathrm{~s}$ edge) and positioned free standing or on a soft X-ray transparent substrate such as $\mathrm{Si}_{3} \mathrm{~N}_{4}$ or thin polymer materials such as polyimide or formvar. Consequently these thin substrates are extremely fragile. We are also limited to patterning relatively small areas. With the current instrument and relatively flat $\mathrm{Si}_{3} \mathrm{~N}_{4}$ substrates the microscope stays in reliable focus over approximately $80 \times 80 \mu \mathrm{m}$. Then there is the issue of write speed. It takes approximately $2-3 \mathrm{~ms}$ for the microscope to accurately position the stages based on interferometer feedback. The shortest period of time the shutter can open and close is about $1 \mathrm{~ms} .1 \mathrm{~ms}$ in the current configuration provides enough dose to bring PMMA into the positive mode regime; thus the speed of patterning in positive mode is not limited by flux. This provides some estimate of the speed of patterning: $10 \%$ of a $20 \times 20 \mu \mathrm{m}$ area with $30 \times 30 \mathrm{~nm}$ pixels can be sufficiently exposed in about 3 minutes. It should be noted that PMMA is not considered to be a very sensitive resist and others are much more sensitive (though their sensitivity to soft X-rays remains to be measured). A faster acting shutter and more sensitive resist would provide some improvement. Many of these issues could be overcome by designing a dedicated $\mathrm{X}$-ray writer instrument optimized for speed with autofocusing capability for patterning large areas (glass microscope slides, Si wafers, etc.). 
Has the time come for such an instrument?

In our opinion, no. Even if the cost and access issues of third generation synchrotrons were addressed by compact, affordable, commercially available X-ray sources with similar performance, we feel it is unlikely that focused soft X-ray lithography will ever compete with electron beam lithography in terms of spatial resolution or throughput. Other groups have investigated zone plate arrays with multiple shutters for high throughput patterning [35, 55, 56]. After noting the difficulty in reproducibly fabricating a single high resolution zone plate, achieving and maintaining the sample to zone plate distance, keeping the order sorting aperture centered, etc., we feel that a large leap in technology will be required to fabricate and manage a zone plate array (tens to hundreds of beams) with parallel performance comparable to this study. Thus it seems that direct write lithography with focused soft X-rays offers negligible advantages for applications currently well covered by electron beam lithography. However one can envisage a number of special and possibly unique applications where focused soft X-ray lithography might be an attractive approach. In our view the biggest advantage is the ability to tune the photon energy. Chemically selective patterning in bilayer [24] and trilayer [25] systems has already been demonstrated. Exploitation of the chemical selectivity will likely drive applications of focused soft $\mathrm{X}$-ray lithography.

\section{Conclusion}

A scanning transmission X-ray microscope has been used to pattern thin films of PMMA and PMGI with $300 \mathrm{eV}$ monochromatic X-rays in a manner analogous to direct write lithography with a focused electron beam. The lithographic characteristics of both polymers were determined over a dose range from 0.1-300 MGy. With optimized doses, $40 \pm 5 \mathrm{~nm}$ developed lines have been produced, which is approaching the diffraction-limited resolution of the zone plate lens used. We believe this to be the smallest feature size created with a zone plate lens at any wavelength in any orientation. The mechanism of the exposure spreading phenomenon was determined to be related to the point-spread function of the zone plate lens. We consider the development of a dedicated X-ray writer instrument to be premature at this point in time. Further investigation using present and future generation STXMs is adequate until there are specific examples which demonstrate unique advantages of focused soft X-ray lithography over existing direct write methods.

Acknowledgements This research was funded by NSERC, CFI and the Canada Research Chairs program. Most of the STXM was carried out at beamline 5.3.2.2 at the ALS, which is supported by the Director of the Office of Science, Department of Energy, under Contract No. DE-AC02-05CH11231. Some results were also obtained at the Canadian Light Source which is supported by the Natural Sciences and Engineering Research Council of Canada, the National Research Council Canada, the Canadian Institutes of Health Research, the Province of Saskatchewan, Western Economic Diversification Canada, and the University of Saskatchewan. We thank Dr. David Kilcoyne and Dr. Tolek Tyliszczak for their expert support of the ALS STXMs and Dr. Jian Wang and Yingshen Lu for their support of the CLS STXM. We are especially gratefully to Professor Ash Parameswaran for his generous gift of PMGI.

\section{References}

1. W.H.F. Talbot, British Patent 565 (1852)

2. M. Hatzakis, J. Electrochem. Soc. 116, 1033 (1969)

3. S.-M. Park, Y.S. Huh, H.G. Craighead, D. Erickson, Proc. Natl. Acad. Sci. USA 106, 15549 (2009)

4. A. Bingham, Y. Zhao, D. Grischkowsky, Appl. Phys. Lett. 87, $051101(2005)$

5. E.W. Becker, W. Ehrfeld, D. Münchmeyer, H. Betz, A. Heuberger, S. Pongratz, W. Glashauser, H.J. Michel, R.V. Siemens, Naturwissenschaften 69, 520 (1982)

6. W. Chao, J. Kim, S. Rekawa, P. Fischer, E.H. Anderson, Opt. Express 17, 17669 (2009)

7. A.D. Wilson, IBM J. Res. Dev. 37, 299 (1993)

8. D.P. Sanders, Chem. Rev. 110, 321 (2010)

9. C.W. Gwyn, R. Stulen, D. Sweeney, D. Attwood, J. Vac. Sci. Technol. B 16, 3142 (1998)

10. G. Möllenstedt, R. Speidel, Phys. Bl. 16, 192 (1960)

11. L. Li, R.R. Gattass, E. Gershgoren, H. Hwang, J.T. Fourkas, Science 324, 910 (2009)

12. V.V. Aristov, in X-ray Microscopy II, ed. by D. Sayre, M. Howells, J. Kirz, H. Rarback (Springer, Berlin, 1988)

13. R.L. Seliger, R.L. Kubena, R.D. Olney, J.W. Ward, V. Wang, J. Vac. Sci. Technol. 16, 1610 (1979)

14. A.E. Brennemann, A.V. Brown, M. Hatzakis, A.J. Speth, R.F.M. Thornley, IBM J. Res. Dev. 11, 520 (1967)

15. G. Schmahl, D. Rudolph, Optik 29, 577 (1969)

16. B. Niemann, D. Rudolph, G. Schmahl, Appl. Opt. 15, 1883 (1976)

17. A.L.D. Kilcoyne, T. Tyliszczak, W.F. Steele, S. Fakra, P. Hitchcock, K. Franck, E. Anderson, B. Harteneck, E.G. Rightor, G.E. Mitchell, A.P. Hitchcock, L. Yang, T. Warwick, H. Ade, J. Synchrotron Radiat. 10, 125 (2003)

18. K.V. Kaznatcheev, Ch. Karunakaran, U.D. Lanke, S.G. Urquhart, M. Obst, A.P. Hitchcock, Nucl. Instrum. Methods Phys. Res., Sect. A, Accel. Spectrom. Detect. Assoc. Equip. 582, 96 (2007)

19. J. Raabe, G. Tzvetkov, U. Flechsig, M. Böge, A. Jaggi, B. Sarafimov, M.G.C. Vernooij, T. Huthwelker, H. Ade, D. Kilcoyne, T. Tyliszczak, R.H. Fink, C. Quitmann, Rev. Sci. Instrum. 79, 113704 (2008)

20. D. Attwood, Soft X-rays and Extreme Ultraviolet Radiation Principles and Applications (Cambridge University Press, Cambridge, 1999)

21. W. Chao, private communication

22. X. Zhang, C. Jacobsen, S. Lindaas, S. Williams, J. Vac. Sci. Technol. B 13, 1477 (1995)

23. R. Larciprete, L. Gregoratti, M. Danailov, R.M. Montereali, F. Bonfigli, M. Kiskinova, Appl. Phys. Lett. 80, 3862 (2002)

24. J. Wang, H.D.H. Stöver, A.P. Hitchcock, T. Tyliszczak, J. Synchrotron Radiat. 14, 181 (2007)

25. J. Wang, H.D.H. Stöver, A.P. Hitchcock, J. Phys. Chem. C 111, $16330(2007)$

26. A.G. Caster, S. Kowarik, A.M. Schwartzberg, S.R. Leone, A. Tivanski, M.K. Gilles, J. Vac. Sci. Technol. B 28, 1304 (2010) 
27. T. Warwick, H. Ade, D. Kilcoyne, M. Kritscher, T. Tyliszczak, S. Fakra, A. Hitchcock, P. Hitchcock, H. Padmore, J. Synchrotron Radiat. 9, 254 (2002)

28. R.W. Johnstone, I.G. Foulds, M.V. Pallapa, A.M. Parameswaran, J. Micro/Nanolithogr. MEMS MOEMS 7, 043006 (2008)

29. A.C. Henry, R.L. McCarley, S. Das, C. Khan Malek, D.S. Poche, Microsyst. Technol. 4, 104 (1998)

30. D.L. Spears, H.I. Smith, Electron. Lett. 8, 102 (1972)

31. J.S. Greeneich, J. Electrochem. Soc. 122, 970 (1975)

32. M. Hatzakis, J. Polym. Sci. 23, 73 (1974)

33. J. Wang, C. Morin, L. Li, A.P. Hitchcock, A. Scholl, A. Doran, J. Electron Spectrosc. Relat. Phenom. 170, 25 (2009)

34. J.S. Greeneich, J. Electrochem. Soc. 121, 1669 (1974)

35. R.E. Burge, M.T. Browne, P. Charalambous, Microelectron. Eng. 6, 227 (1987)

36. R. Klauser, I.-H. Hong, S.-C. Wang, M. Zharnikov, A. Paul, A. Gölzhäuser, A. Terfort, T.J. Chuang, J. Phys. Chem. B 107, 13133 (2003)

37. A.D. Dubner, A. Wagner, J.P. Levin, J. Mauer, J. Vac. Sci. Technol. B 10, 3212 (1992)

38. G. Compagnini, G.G.N. Angilella, A. Raudino, O. Puglisi, Nucl. Instrum. Methods Phys. Res., Sect. B, Beam Interact. Mater. Atoms 175-177, 559 (2001)

39. M. Born, E. Wolf, Principles of Optics, 7th edn. (Cambridge University Press, Cambridge, 1999)

40. M.D. Galus, E. Moon, H.I. Smith, R. Menon, J. Vac. Sci. Technol. B 24, 2960 (2006)

41. A.N. Broers, IBM J. Res. Dev. 32, 502 (1988)

42. A.N. Broers, A.C.F. Hoole, J.M. Ryan, Microelectron. Eng. 32, 131 (1996)
43. M.P. Seah, W.A. Dench, Surf. Interface Anal. 1, 2 (1979)

44. S. Yasin, D.G. Hasko, H. Ahmed, Appl. Phys. Lett. 78, 2760 (2001)

45. W. Hu, K. Sarveswaran, M. Lieberman, G.H. Bernstein, J. Vac. Sci. Technol. B 22, 1711 (2004)

46. E. Lavallée, J. Beauvais, J. Beerens, J. Vac. Sci. Technol. B 16, 1255 (1998)

47. N. Arjmandi, L. Lagae, G. Borghs, J. Vac. Sci. Technol. B 27, 1915 (2009)

48. G. Schmahl, D. Rudolph, D. Neimann, in Proc. Eight Int. Conf. on X-ray Optics and Microanalysis, Boston (1977), p. 60A

49. S. Heim Rehbein, P. Guttmann, S. Werner, G. Schneider, Phys. Rev. Lett. 103, 110801 (2009)

50. H. Mimura, S. Matsuyama, H. Yumoto, Jpn. J. Appl. Phys. 44, L539 (2005)

51. C.R.K. Marrian, E.A. Dobisz, J.A. Dagata, J. Vac. Sci. Technol. B 10, 2877 (1992)

52. P.A. Peterson, Z.J. Radzimski, S.A. Schwalm, P.E. Russell, J. Vac. Sci. Technol. B 10, 3088 (1992)

53. W. Chao, B.D. Harteneck, J.A. Liddle, E.H. Anderson, D.T. Attwood, Nature 435, 1210 (2005)

54. A.P. Hitchcock, J.J. Dynes, G. Johansson, J. Wang, G. Botton, Micron 39, 741 (2008)

55. H.I. Smith, J. Vac. Sci. Technol. B 14, 4318 (1996)

56. A. Pépin, D. Decanini, Y. Chen, J. Vac. Sci. Technol. B 18, 2981 (2000) 